\title{
Escape Path Obstacle-Based Mobility Model (EPOM) for Campus Delay-Tolerant Network
}

\author{
Sirajo Abdullahi Bakura ${ }^{D}$, Alain Lambert, and Thomas Nowak \\ Université Paris-Saclay, CNRS, Laboratoire Interdisciplinaire des Sciences du Numérique, 91405 Orsay, France \\ Correspondence should be addressed to Sirajo Abdullahi Bakura; sirajo.abdullahi-bakura@universite-paris-saclay.fr
}

Received 21 June 2021; Revised 26 October 2021; Accepted 30 November 2021; Published 30 December 2021

Academic Editor: Eleonora Papadimitriou

Copyright ( 2021 Sirajo Abdullahi Bakura et al. This is an open access article distributed under the Creative Commons Attribution License, which permits unrestricted use, distribution, and reproduction in any medium, provided the original work is properly cited.

\begin{abstract}
In Delay-Tolerant Networks (DTNs), humans are the main carriers of mobile devices, signifying that human mobility can be exploited by extracting nodes' interests, social behavior, and spatiotemporal features for the performance evaluation of DTNs protocols. This paper presents a new mobility model that describes students' daily activities in a campus environment. Unlike the conventional random walk models, which use a free space environment, our model includes a collision-avoidance technique that generates an escape path upon encountering obstacles of different shapes and sizes that obstruct pedestrian movement. We evaluate the model's usefulness by comparing the distributions of its synthetic traces with realistic traces in terms of spatial, temporal, and connectivity features of human mobility. Similarly, we analyze the concept of dynamic movement clusters observed on the location-based trajectories of the studied real traces. The model synthetically generates traces with the distribution of the intercluster travel distance, intracluster travel distance, direction of movement, contact duration, intercontact time, and pause time similar to the distribution of real traces.
\end{abstract}

\section{Introduction}

Mobility patterns play an essential role in the performance of wireless networks with intermittent connections such as Delay-Tolerant Networks (DTNs). Some of the features associated with these networks include persistent disconnections, absence of simultaneous end-to-end communication routes, sparse topology, long delays among nodes due to mobility, and sparse deployment of nodes. However, we can achieve a weak form of connectivity in DTNs by exploiting the temporal dimension and node mobility [1]. Considerable research efforts have been put recently to enable communications between network entities with intermittent connectivity [2].

Moreover, the forwarding opportunities of the DTNs depend on the patterns of mobility that dictate contact opportunities between nodes for reliable information forwarding. Interestingly, humans are the main carriers of mobile devices. Therefore, there is a need to understand the underlying behavior of pedestrian mobility, the driving forces that influence its motivation to move, and the repulsive forces that describe its interaction with environmental constraints. These are essential for designing a realistic mobility model to be used as a tool for wireless network protocol evaluation, hence the need for a model based on the empirical study of pedestrians' mobility and interaction with other objects in the environment to pave the way for better event management, emergency rescue operation, and congestion prediction in a narrow bottleneck.

This study investigated the mobility characteristics of pedestrians using real traces and proposed an obstacle-based mobility model for the DTNs that closely replicates the empirical features observed in the analyzed traces and generates spatial, temporal, and connectivity features similar to the features generated by realistic human mobility to enhance opportunistic forwarding for the DTNs and support pedestrian collision avoidance in the event of crowd or emergency rescue operations.

Several mobility models have been proposed in [3-10], which can be categorized into synthetic or trace-based. The 
synthetic mobility models are less realistic as compared to the trace-based ones. On the contrary, the trace-based models are much more difficult to develop than the synthetic models. In addition to the models that describe pure human mobility, the models presented in [11-13] have explored the concept of cognitive science modeling using the driving forces that influence pedestrians' internal motivation to move in a given direction and speed and the repulsive forces that describe pedestrian interaction with other pedestrian and environmental constraints such as obstacles using empirical data obtained from laboratory-controlled experiments. In this regard, we concentrate on the pedestrians' interaction with the static and moving obstacles.

Starting with the conventional mobility models, random walks are the most widely used synthetic models for the analysis of node mobility [3, 4]. Random walk models generate mobility patterns in which mobile nodes display completely random behavior. With this regard, only a few wireless networks (e.g., sensor network for animal tracking $[14,15])$ can display such kind of randomness. In contrast, the majority of wireless networks strictly obey certain mobility rules. Pedestrian mobility is not completely random but influenced by features specific to humans, which resemble intentional mobility toward points of attraction.

On the contrary, the random waypoint model $[8,16,17]$ is considered the first synthetic model that attempts to model intentional human movement, which is not captured in the random walk models. Nevertheless, the model was shown to be unrealistic in [9] due to its failure to provide a steady state, resulting in the inconsistent decrease of an average node speed over time.

This property can lead to unreliable results. Some simple fixes and modifications to the random waypoint presented in [9] still fail to capture the realistic behavior of intentional human mobility to some locations due to the strength of a social relationship or connection. For instance, a student might go to class for the lecture and to the cafeteria to eat or visit a nearby dormitory friend.

The node's movement is not restricted to a pathway in the random walk and random waypoint models. The Manhattan mobility model [18], on the contrary, restricts the movement of a node to the pathway in the simulation area.

A generalization of several classical models that attempt to develop synthetic mobility models for mobile networks and satisfy some statistics was presented in $[6,7,19,20]$. Some of the statistical features studied are the flight distribution (i.e., a straight line distance covered between two consecutive waypoints), pause time distribution (i.e., the amount of time a node pauses at a waypoint), and intercontact time distribution (i.e., the amount of time between two contacts of the same pair of nodes) regarding different scales of time and space.

To develop mobility models using empirical data, which is the concept adopted in our study, studies in [21-23] extract detailed mobility data from real traces and calibrate the uncovered mobility features in their models. The studies in $[21,22]$ recognize opportunities when the user is associated with the same Wi-Fi access point; the idea was extended in Kim et al. [23] by considering a situation when the users are within the communication range of each other. Kim et al. [23] proposed a synthetic mobility model from user mobility characteristics extracted from the wireless network traces syslog but only considers Wi-Fi access points, which have a higher granularity of mobility trajectories. In [11], a controlled laboratory experiment was conducted to study the behavioral effects of interactions between pedestrians. The study extracts individual behavioral laws from the statistical features observed in the empirical data.

The contributions of this paper are threefold:

(1) We conduct a characterization of spatial, temporal, and connectivity features of human mobility using real traces.

(2) We conduct an in-depth study on the movement displacements and directions within the movement clusters characterized by short walks within a confined area.

(3) We propose an Escape Path Obstacle-based Mobility Model (EPOM) for the campus DTNs. We show that the model is generic enough to be fine-tuned with a few parameters to show matching characteristics with the spatiotemporal and connectivity features observed in the real traces.

This paper is organized as follows: Section 2 presents a review of the related works. Section 3 explains the characterization of human mobility. Section 4 describes the Escape Path Obstacle-based Mobility Model and its submodels. Section 5 describes the implementation of the proposed model. Section 6 presents the settings and the results of the simulations. Finally, the conclusion and future perspectives are given in Section 7 .

\section{Related Work}

The increasing interest of the research community on the DTNs and the impacts of mobility on their performance has led to the development of several mobility models focusing on different mobility features $[6,7,19,20,24]$. Nevertheless, the conventional synthetic stochastic models $[3,4,9,25,26]$ meant for the performance analysis of network protocols in the early ad-hoc networks are insufficient to capture users' intentional behaviors and social attractions. Several works have investigated the adaptability of the conventional models in the next generation mobile networks such as DTNs, Vehicular Ad-hoc Networks (VANETs), and Wireless Sensor Networks (WSNs). The studies found that human mobility is characterized by intentional mobility as opposed to the random assumptions in the conventional models [7, 19, 20].

Although synthetic models that capture intentional human behavior are more realistic than the conventional models, the trace-based models [22, 27] appear to be more realistic because they are mostly generated for a specific scenario and only for a few nodes. In contrast, the nonconventional synthetic models $[7,19,20]$ can generate synthetic mobility traces for a large number of nodes 
considering mobility constraints such as obstacles and pathways. The generated traces are used to evaluate network protocols.

In this regard, an in-depth understanding of the interaction between pedestrians and pedestrians with other obstacles in a realistic domain aids in simulating emergency scenarios for pedestrian safety [11-13].

Lee et al. [6] employed the concept of fractal waypoints, Least Action Trip Plan (LATP), and a walker model to generate regular patterns of daily human mobility. Their model is based on daily routine activities such as going to the office or attending a lecture. However, the model did not capture an event's occurrence time and the repetitiveness observed in the people's realistic daily activities.

Munjal et al. [7] presented a mobility model that mimics real human mobility patterns by relaxing an assumption of random mobility with a notion of a mobility influence; that is, nodes mobility is influenced by factors such as cluster size. The model studies seven mobility statistical features: the flights, intercontact time, pause time, long flight due to popularity, closest mobile node visits, community interaction, and mobile node distribution. However, the simulation space in Munjal et al. [7] is a free space without restricting obstacles, which is not always realistic in a real environment such as a campus setting characterized by buildings of different shapes and sizes.

In an attempt to develop a mobility model that captures people's agendas or activities, several models were developed [19, 20, 24, 28, 29]. Ekman et al. [19] presented a Working Day Model (WDM) that emulates the workers' daily activities such as going to the office, going for evening activities, or returning home. The model uses map-based movement on the concept of sources destination. It also uses a timescale to switch between different submodels. Ekman et al. [19] showed the similarities between the distribution of the synthetic traces of his model and that of iMotes traces from the Cambridge experiment. However, they did not cover the impacts of obstacles, such as the floor, walls, and other constraints, which affect nodes' mobility.

In [29], the characteristics of human mobility were described by constructing multidimensional mobility space, divided into individuality metrics, pairwise encounter metrics, and group metrics. The model generates node trajectories that show more human mobility characteristics, but it was validated using a conventional model, that is, the random waypoint mobility model.

Students' daily activities on the campus were studied by Zhu et al. [20] with a focus on the contact time, intercontact time, and contact per hour distributions. This work did not consider the impacts of obstacles in restricting the free movement of the mobile nodes and a possible signal obstruction by buildings of different shapes and sizes in the campus environment.

Social mobility models were presented in $[30,31]$. Hrabcak et al. [24] presented a Students Social Based Mobility Model (SSBMM). Their work was inspired by the daily routine of a student's life. The model distinguishes between the student's free time and the mandatory time upon which social and school activities are simulated. They compare their model with the classical random walk model, even though the random walk model cannot capture the repetitiveness and heterogeneity of time and space in human mobility.

Wang et al. [32] proposed an obstacle-based mobility model that generates a smooth trajectory of a Bezier curve for escaping obstacles. Human mobility trajectories for escaping obstacles such as building or road diversion are not always smooth curves in real scenarios. In addition, the model did not capture movement to attraction factors such as points of interest, which represents human social behavior.

The Obstacle Mobility (OM) model developed by Jardosh et al. [33] models the environmental obstructions which affect both movement and signal propagation. In this model, the node paths and points are constructed from a Voronoi diagram based on the obstacle position on the campus-like simulation area.

As an extension to [33], Papageorgiou et al. [34] proposed a model that allows nodes to move around the obstacle but is not limited to a defined path. The model considers rectangular obstacles that limited its ability to capture the realistic feature of an environment with obstacles of different shapes and sizes.

A random obstacle-based mobility model for DTN was presented by $\mathrm{Wu}$ et al. [28]. In this model, the node moves from the initial location to the destination via the shortest path if there is no obstacle along the path; otherwise, the node recursively selects the node's location close to the obstacle and moves forward. This operation is repeated until the node reaches its destination. This model considers obstacles with a rectangular shape. Similarly, an unnecessary trip would be made in the absence of a node close to the obstacle, especially when the destination is just behind the obstacle.

Wang et al. [32] proposed an obstacle-based mobility model that generates a smooth trajectory of a Bezier curve for escaping obstacles. Human mobility trajectories for escaping obstacles such as building or road diversion are not always smooth curves in real scenarios. In addition to that, the model did not capture movement to attraction factors such as points of interest that represent human social behavior.

Moussaid et al. [11] conducted an experimental study of the behavioral mechanisms underlying self-organization in human crowds to study an individual pedestrian behavior. In the study, an individual pedestrian movement behavior is characterized by the triplet: the internal acceleration $f_{i}^{0}$, wall interaction $f_{i}^{\text {wall }}$, and individual interaction $f_{i j}$. To simplify the complexity of the assumptions, a study that uses simple rules to determine pedestrian behavior and crowd disasters was presented in [12]. The study used simple heuristics to determine the movement direction and possible choice of desired speed during static and moving obstacle encounters.

Some of the research works in the literature have studied mobility characteristics in real traces to develop synthetic mobility models that exhibit the observed mobility features in the real traces. Kim et al. [23] analyzed mobility characteristics, including pause time, speed, and direction of 
movements and developed a software model that generates realistic user mobility tracks but the mobility trajectory granularity of the studied trace depends on the wireless Local Area Network (WLAN) access point locations and hence may not be applicable to higher mobility DTN.

Real mobility traces at Dartmouth College [35] and Disney World theme park in Orlando [36] have been analyzed in [37] to obtain movement characteristics. Consequently, they obtained a visiting probability of people, distribution of movement speed, and pause time from the traces. Their proposed model is configured with the derived distribution in the simulation.

However, several works have proposed different techniques for mining mobility patterns or mobility behaviors from the trajectory data.

Ghosh et al. [38] proposed a mobility pattern mining framework to extract mobility association rules from taxi trips. The proposed framework has three modules: the input module, the spatiotemporal analysis module, and the mobility association generation module. The input module processes the Taxi GPSlog, road network, and Point-OfInterest and generates transactions using application-specific mobility rule templates. The spatiotemporal analysis model analyses travel demand data and partition regions based on the travel demand and then generates mobility flow. Lastly, the mobility association generation module delineates how it can be used to understand urban dynamics.

Yue et al. [39] proposed a trajectory clustering technique for mobility-behavior analysis. In their approach, they formulate mobility analysis as a clustering task. They developed an unsupervised learning technique that resolves the problem of lack of labeled trajectory data that support supervised learning, in which data does not necessarily need to be labeled.

Rahman et al. [40] presented a dynamic clustering technique based on the processed COVID-19 infection data and mobility data. In this work, clusters can expand and shrink based on the merit of the data.

This study presents an Escape Path Obstacle-based mobility Model (EPOM) for a campus Delay-Tolerant Network. Our model covers aspects such as daily routines, heterogeneity of time and space, skewed location visiting, and the discovered dynamic cluster evolution. We also develop a novel strategy for collision avoidance between pedestrians and obstacles of different shapes and sizes. Our model mimic more realistic behavior observed in the realistic traces.

Table 1compares existing works and the EPOM mobility model in terms of the most widely studied mobility features and the evaluation methods. Symbol $X$ indicates that the existing work studied the mobility feature while symbol $x$ indicates the opposite.

\section{Characterization of Human Mobility}

Several efforts have been devoted to investigating the properties of human mobility and uncovering hidden patterns $[23,41,42]$. Due to the dynamic nature of human mobility, there is no consensus on its characteristic features.
The features that require a thorough investigation include but are not limited to some of the fundamental features such as travel distance and pause time. However, there is a need to understand the features of a movement cluster within the community, such as the intracluster travel distance and direction of movement, though we explicitly study some fundamental features for the whole domain: the connectivity features (i.e., contact and intercontact time), the spatial feature (i.e., travel distance), and the temporal feature (i.e., pause time). The movement direction within the clusters, to the best of our knowledge, has been assumed to be random [41] or reported for the entire domain [23, 42].

In this study, we use daily GPS track log collected from two different university campuses (NCSU and KAIST) for the location-based trace [36]. Garmin GPS 60CSx handheld receivers are used for data collection which are WAAS (Wide Area Augmentation System) capable with a position accuracy of better than three meters 95 percent of the time, in North America. The GPS receivers take reading of their current positions at every 10 seconds and record them into a daily track log. The data are available at [43]. We are interested in the stationary locations at which users stay.

For the contact-based trace, we use the Bluetooth encounters between mobile nodes from the Cambridge city students iMote experiment [44]. The data consist of 10641 contacts between iMote devices carried by students for the duration of about 11.43 days. The data is available at [43] repository. We are interested in the duration at which two devices are in contact with each other (contact duration) and the time between two consecutive contacts between two devices (intercontact time).

We emphasize that the cluster concept in our study refers to the location at which a person spends much of his time exploring the neighboring locations. Therefore, it should not be confused with the concept in a social community, which refers to people sharing physical location, ideas, or common goals. A person can generate more than one cluster within his community, depending on his daily trip schedules. Figure 1 shows the trajectory of user 16 in the KAIST trace, creating four dynamic clusters for one day. In our clustering, we consider only clusters that have more than eight locations within a specified threshold.

Before clustering, we have to remove transit locations from our traces, which is reasonable because some of the coordinates from the GPS traces do not belong to stationary locations; they belong to the transit locations at which a user stays briefly on its way to its destination. Algorithm 1 summarizes the procedures for removing transit locations. In Algorithm 1, point $p_{i+1}$ is deleted if the distance between $p_{i}$ and $p_{i+1}$ is greater than a distance threshold. Similarly, if the pause time at point $p_{i}$ is less than a time threshold, point $p_{i}$ is removed from the original trace. When Algorithm 1 is executed, our original trace would be left with only stationary stations.

Next, we run an agglomerative clustering technique [45] using a single linkage method, sometimes called connectedness or minimum method and created location clusters based on the similarity of the closest pair of locations. 
TABLE 1: Comparison of existing works and the EPOM mobility model in terms of most widely studied mobility features.

\begin{tabular}{|c|c|c|c|c|c|c|c|c|}
\hline Features & [20] (2012) & [19] (2008) & [7] (2011) & [9] RWP & [37] (2020) & [34] (2009) & [34] (2017) & EPOM \\
\hline Obstacle-aware & $\mathrm{x}$ & $\mathrm{X}$ & $\mathrm{X}$ & $\mathrm{x}$ & $\mathrm{X}$ & $\checkmark$ & $\checkmark$ & $\checkmark$ \\
\hline Obstacle-shape & $\mathrm{x}$ & $\mathrm{X}$ & $\mathrm{x}$ & $\mathrm{x}$ & $\mathrm{X}$ & $\begin{array}{c}\text { Rectangular } \\
\text { shape }\end{array}$ & $\begin{array}{l}\text { Irregular } \\
\text { shape }\end{array}$ & $\begin{array}{l}\text { Irregular } \\
\text { shape }\end{array}$ \\
\hline Travel distance & $\mathrm{X}$ & $\checkmark$ & $\checkmark$ & $\mathrm{X}$ & $\mathrm{X}$ & $\mathrm{X}$ & $\mathrm{X}$ & $\boldsymbol{J}$ \\
\hline Direction of movement & $\mathrm{x}$ & $\mathrm{X}$ & $\mathrm{x}$ & $\mathrm{x}$ & $\mathrm{x}$ & $\mathrm{X}$ & $\mathrm{X}$ & $\boldsymbol{J}$ \\
\hline Pause time & $\mathrm{x}$ & $\checkmark$ & $\checkmark$ & $\mathrm{x}$ & $\checkmark$ & $\mathrm{X}$ & $\mathrm{X}$ & $\checkmark$ \\
\hline $\begin{array}{l}\text { Contact time and } \\
\text { intercontact time) }\end{array}$ & $\checkmark$ & $\checkmark$ & $\checkmark$ & $\mathrm{x}$ & $\mathrm{x}$ & $\mathrm{X}$ & $\mathrm{x}$ & $\checkmark \checkmark x$ \\
\hline Evaluation method & Real traces & Real traces & Real traces & $\begin{array}{c}\text { Real } \\
\text { traces }\end{array}$ & Real traces & $\begin{array}{l}\text { Obstacle } \\
\text { simulation }\end{array}$ & $\begin{array}{l}\text { Obstacle } \\
\text { simulation }\end{array}$ & $\begin{array}{c}\text { Real traces } \\
\text { and } \\
\text { obstacle sim }\end{array}$ \\
\hline
\end{tabular}

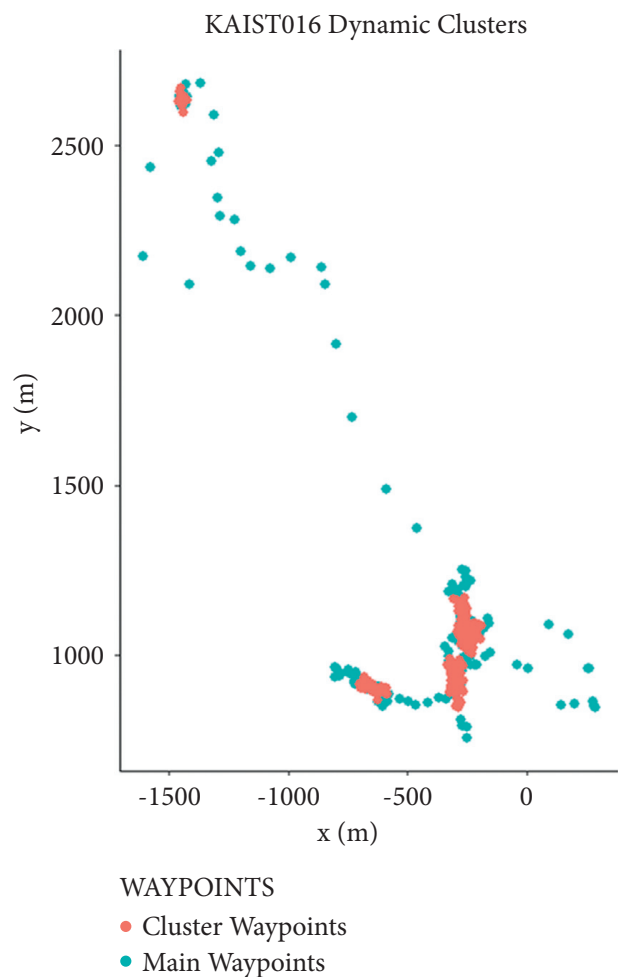

FIgURE 1: The dynamic clusters of KAIST trace file 16. The blue points indicate the complete waypoints in a day, while the red points indicate the waypoint clusters. There are four clusters associated with the user.

Initially distanceThreshold $=\Delta_{d}$, waitingTimeThreshold $=\Delta_{t}$, First Point $=P_{i}$, Second Point $=P_{i+1}$.

(1) if distance $\left(P_{i}, P_{i+1}\right) \geq \Delta_{d}$ then

(2) remove $P_{i+1}$

(3) if pauseTime $\left(P_{i}\right) \leq \Delta_{t}$ then

(4) remove $P_{i}$

Algorithm 1: Extracting dynamic clusters from the location-based traces. 
The more we know about the movement cluster properties such as a travel distance and direction of movement, the more we can predict the behavior of a pedestrian movement pattern for accurate design against possible crowd congestion or emergency scenarios.

3.1. Intracluster Direction of Movement. The direction of movement within the movement clusters has not been studied well despite its impacts on the mobility patterns. Nevertheless, some related works reported an aggregate distribution of movement directions for the whole domain instead of movement clusters [41, 42]. Our study takes a different approach; it studies the direction of movement within dynamic clusters to understand the direction angle's properties.

Figure 2 shows a weighted Probability Density Function (PDF) of the movement direction within clusters from NCSU trace with a bin size of $1^{\circ}$. We measure the direction of each movement by its movement duration. We can see that the direction of movement is biased symmetric toward some preferred locations. It implies that the movement within a dynamic cluster is not random; it favors directions of the popular locations. The symmetry distribution was expected due to the possible return of nodes to their main locations after exploring some close by points of interest. We can also deduce that students visit common locations for their activities, which resulted in the similar aggregated distribution of angles with bias symmetry to angles between $90^{\circ}-150^{\circ}$ and $240^{\circ}-330^{\circ}$, respectively. We can see that nodes move to other sides as well, but with smaller frequencies than the direction of the point of interest; this implies that geographical restrictions such as constraint movement on roads are not the driving factor for the bias symmetry of the movement angle distribution. On the contrary, the aggregate weighted PDF for the whole domain is shown in Figure 3. Figure 3 shows that though it has a symmetry shape, the direction of movement is almost uniformly distributed within the domain.

3.2. Intracluster Travel. We study the travel distances between consecutive locations within the cluster at which a node spent a long time exploring neighboring locations.

We fit four parametric models on the empirical data of KAIST intracluster travel distance as shown in Figure 4. The distribution that best fits the data is the lognormal distribution with the parameters 2.29989493 and 0.8685148 for the log mean and log standard deviation, respectively, as shown in Figure 5 and KS test in Table 2. This shows that students take repeated short walks around some popular locations such as classes, libraries, and dormitories.

3.3. Pause Time Distribution. Pause time distribution is one of the temporal features of human mobility, which plays a vital role in the diffusive nature of human mobility. It dictates the amount of time a node spends at a location with zero or close to zero velocity. Figure 6 shows four different parametric models that fit the empirical data of the KAIST

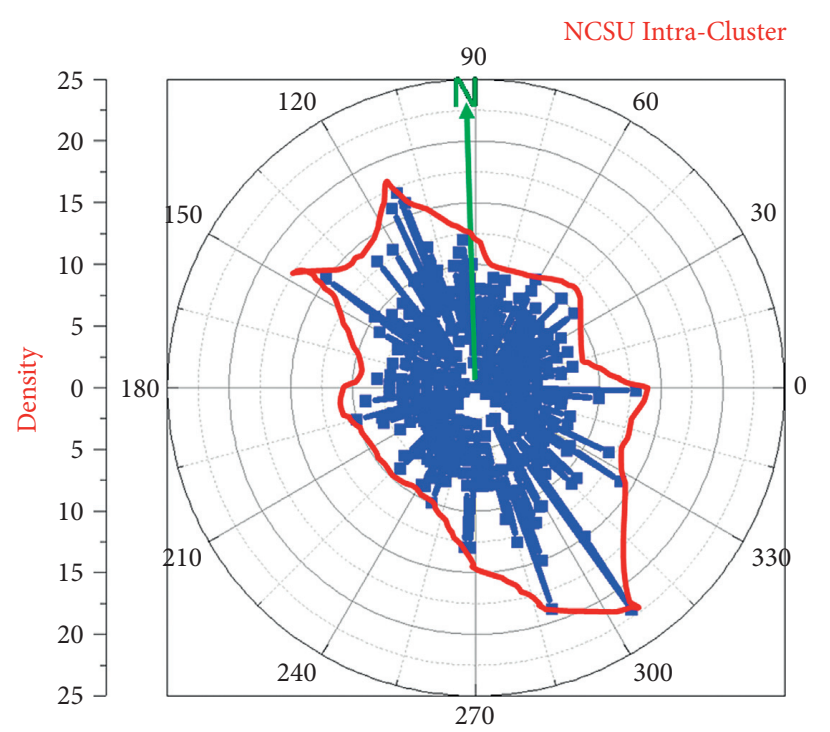

FIgURE 2: The bias symmetry distribution of direction angle for the dynamic clusters (NCSU traces). The $x$-axis represents the angular (units are in degrees), and the $y$-axis is the density of movement toward a given direction. The bin size is $1^{\circ}$. Each direction is weighted by the duration of its movement.

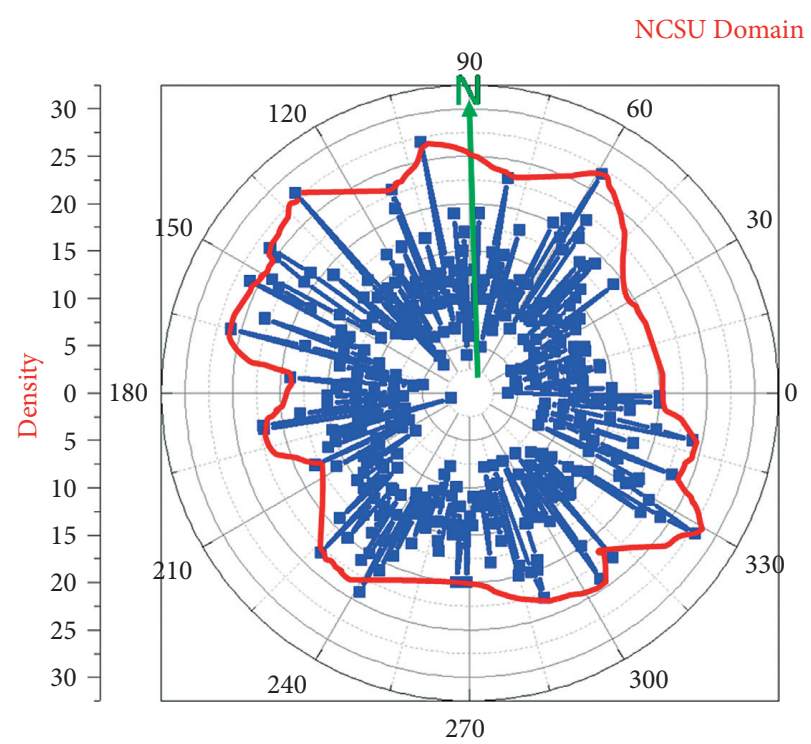

FIgure 3: The uniform distribution of direction angle for the whole domain (NCSU traces). The $x$-axis represents the angular (units are in degrees) and $y$-axis is the density of movement toward a given direction. The bin size is $1^{\circ}$. Each direction is weighted by the duration of its movement.

trace. After the KS test of gof, we found that power-law distribution is plausible, and hence there is no enough evidence to support its rejection as shown in Table 3. Figure 7 shows that the power law has a threshold value (xmin) of four minutes $(240 \mathrm{~s})$ and a cut-off value of $P(\Delta(t))=16 \mathrm{hrs}$. The power-law pause time distribution indicates a scale-free characteristic. 


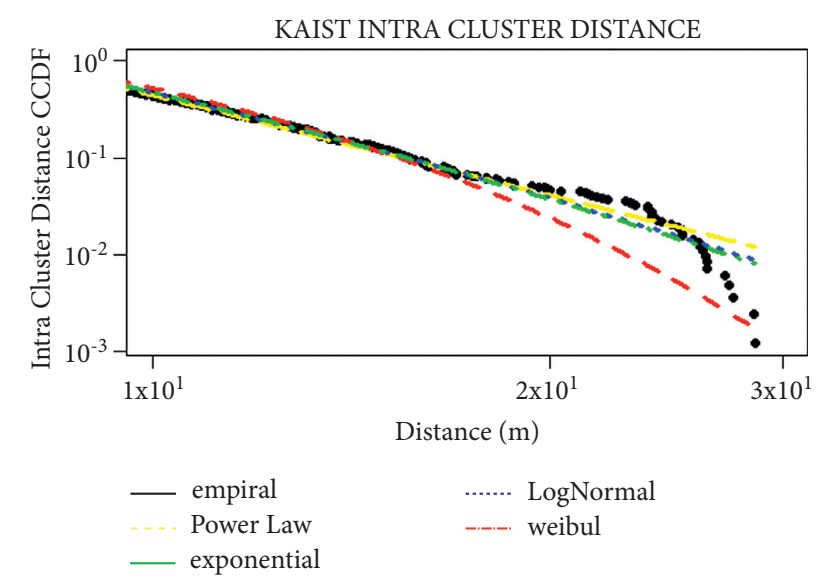

FIgURE 4: Four (4) different distributions fitted to the KAIST intracluster distance trace; all distribution fits the empirical data, but power law and lognormal show better matching features.

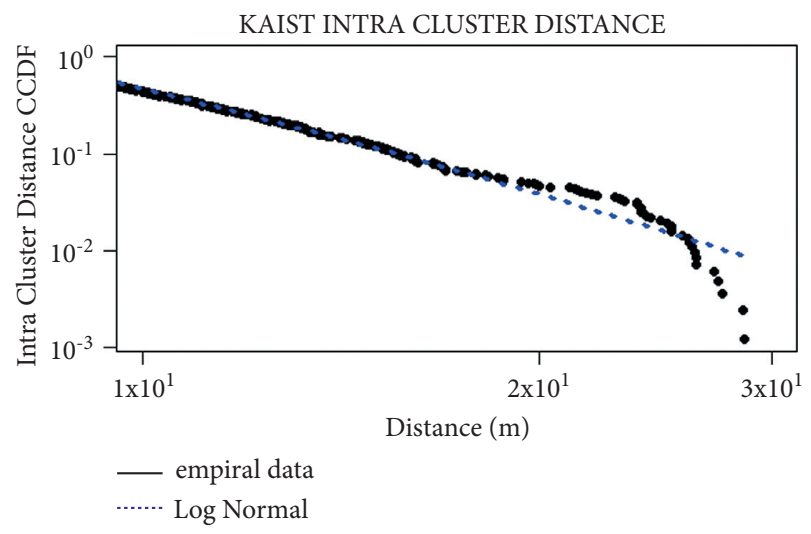

Figure 5: Log-normal fits the KAIST intracluster.

TABLE 2: KAIST intracluster distance gof table.

\begin{tabular}{lcccc}
\hline Dist & gof & Ntails & Crit. Val & Remark \\
\hline Lognorm & 0.01564494 & 815 & 0.04764 & Accept \\
Power law & 0.03181678 & 1900 & 0.03120 & Reject \\
\hline
\end{tabular}

3.4. Intercontact Time. In this section, we characterize the empirical data from iMotes experiments at Cambridge [44]. The data includes some traces of Bluetooth sightings by groups of users carrying small devices (iMotes) for five days. Our goal is to extract the distribution of intercontact time from the dataset for further analysis. Figure 8 shows the aggregate CCDF distribution for the intercontact duration of the empirical data. The distribution follows a power-law distribution with the exponent $=1: 4$, but the power-law decay is overweight by an exponential decay toward the end of the distribution. The distribution is called a truncated power law, similar to the results presented in [6]. The power feature of the intercontact time distribution is interesting because it dictates the scale-free properties of an opportunistic network.

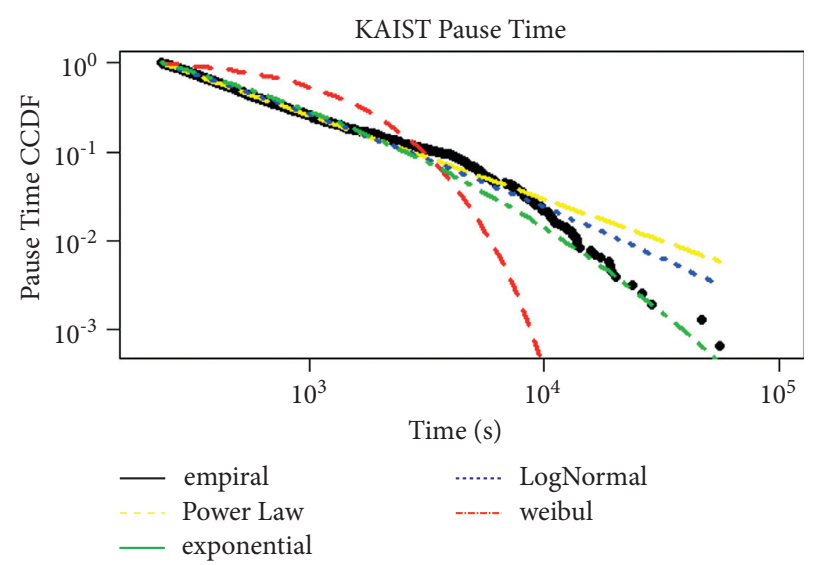

FIgURE 6: Four (4) different distributions fitted to the KAIST trace pause time.

TABLE 3: KAIST pause time gof table.

\begin{tabular}{lcccc}
\hline Dist & gof & Ntails & Crit. value & Remark \\
\hline Power law & 0.02367702 & 850 & 0.04665 & Accept \\
Exponential & 0.1315407 & 386 & 0.06922 & Reject \\
\hline
\end{tabular}

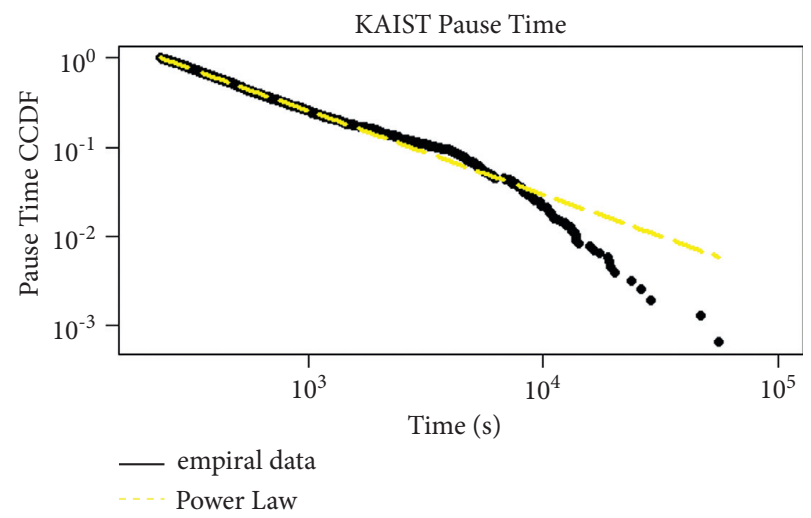

Figure 7: A pause time distribution for the KAIST trace. The distribution exhibits power-law decay with exponential cut-off.

\section{EPOM Model}

EPOM was developed by integrating several submodels or communities into one functional model. Each submodel captures a specific, realistic activity of a campus environment as observed in the empirical data of the studied traces [36, 44]. We have categorized the realistic activities into the home, study, eating, sports, and off-campus activities, respectively. In addition to the submodels, a switching model plays an important role in switching the status of nodes between the submodels; this is referred to as intercluster movement. It helps to capture the realistic nature of students' lives on a campus, which is viewed as repetitive and heterogeneous in time and space. When a node changes its status (e.g., from home to study), it uses the preferred method of transport to move to its new destination. We adopted two methods of transport in the model: walking and bus. 


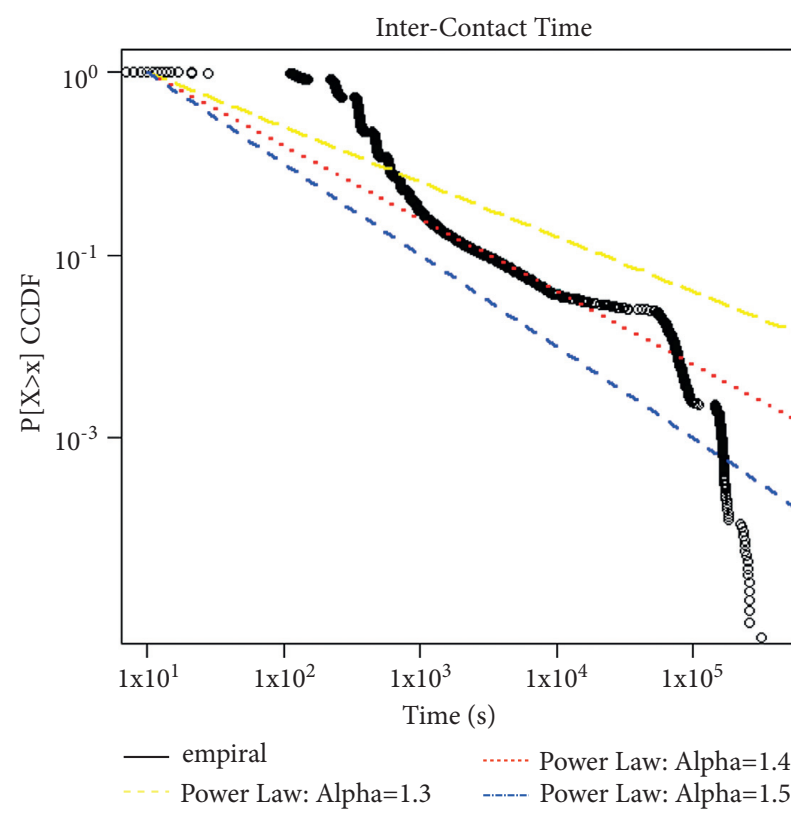

FIGURe 8: Power-law distribution with different values of lambda fitted on the Cambridge iMotes trace intercontact time distribution.

The EPOM model captures personnel (e.g., faculty/departmental staff) movement, such as walks to the cafeteria for eating, and off-campus activities such as shopping.

One of the distinguishing features of EPOM from the previous works in $[7,8,19,20]$ is the consideration of static and moving obstacles along the movement path from the source to the destination. The EPOM ensures collision-free movement along the trajectories. We model this by strategically placing objects of different shapes and sizes on the movement trajectories and developing an algorithm that generates detour paths. This is reasonable because there is a need for a path to escape a non-moving pedestrian on the movement path. The pictorial description of different submodels is depicted in Figure 9.

4.1. Home Submodel. Home is the starting point of the simulation. Initially, a predefined location is assigned to each node in the home location file. These locations are used for sleeping or node's free time. Daily routine activities of a node start in the morning when it wakes up from the sleeping state. Each node is assigned a wake-up time, which determines when the node should wake up from sleeping. The wake-up time obeys a normal distribution with the mean seven o'clock and configurable standard deviation.

After waking up, a node checks its lecture schedules and decides whether to go for a lecture or do some in-home activities such as cooking, watching the morning news, laundry services, or visiting a friend at the nearby dormitory. These short walks account for the possible evolution of the first dynamic cluster. Some nodes leave their home without doing any internal activities. Depending on the time of the day and a node lecture schedules, a node can switch to other submodels from home. For example, a node may switch to the sport submodel in the evening to play games; it can switch to eating submodel for dinner or switch to offcampus submodel for shopping or visiting a friend in another location. This flexibility of EPOM captures social influence and heterogeneity in time and space.

4.2. Study Submodel. We assigned specific locations on the map as lecture rooms. If a node is in the lecture room, it walks within the lecture room and pauses for the lecture duration. The pause time distribution is location-dependent in our model. The pause time for the lecture is different from the pause time at the cafeteria. However, the pause time at nonspecific locations is derived from the truncated powerlaw distribution observed in the empirical data. We turn off the pause time completely during the lecture period for 80 percent of the nodes; only 20 percent can make some movement within the lecture room; this is to capture the realistic behavior of students for changing desks or forming discussion groups. At the end of the lecture, a node decides to walk to the laboratory or library. This internal movement is modeled as an intracluster walk within the vicinity of the study area with the libraries, laboratories, and other studyrelated locations as waypoints.

4.3. Eating Submodel. Some strategic locations on the map are defined as cafeterias. When it is time for lunch or dinner, a node may switch to eating submodel and move to the cafeteria to eat. The time distribution for eating is uniformly distributed, starting from 11:00 a.m. to 2:00 p.m. for lunch and 6:00 p.m. to 8:00 p.m. for dinner. While in the cafeteria, a node waits, makes some intracluster walks, gets served, then eats, and switches to another submodel. During the eating activities in a large cafeteria, we observed a large crowd of students within a confined location, hence, the need for collision avoidance to allow smooth flow of students.

4.4. Sport Submodel. We define some points on the map as playgrounds; the time for sport is also defined. A node in the sport submodel spends some time at the playground watching or doing some random intracluster movements around the vicinity of the playground.

4.5. Off-Campus Submodel. The off-campus submodel models all activities not included in the home, school, eating, and sport submodels. These activities include shopping, evening walk, or visiting friends. We define some points of interest (PoIs) on the map edges as meeting points. We have two types of PoIs: location preferences PoI and Bus Normal PoI with uniform preferences. Mobile nodes visit such locations in a group to capture group mobility characteristics and social influences of human mobility and individually to capture independent mobility freedom. The minimum and maximum sizes of a group are defined in the default setting file. 


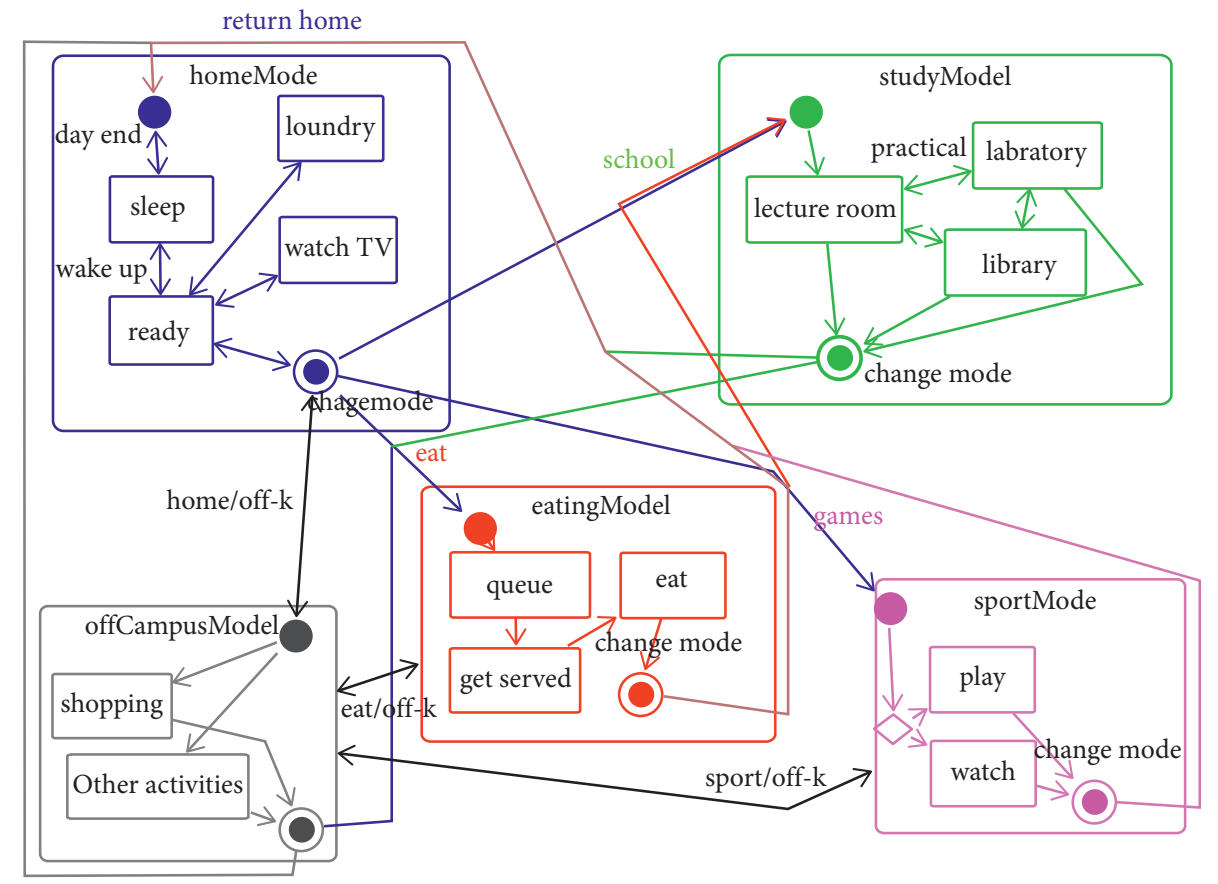

Figure 9: EPOM submodels: the large rectangles represent the submodels (i.e., home, study, cafeteria, sport, and off-campus). The line connecting them is a transport submodel. The red shapes are obstacles that affect both mobility and signal propagation.

4.6. Transport Submodel. This submodel is used to move between different submodels when a node switches mode. We define two means of transport in our model: walking and bus riding. Most nodes walk while a bus is mostly used for off-campus activities. The probability of moving with a bus is configured in the setting. The heterogeneity in the transport submodel has a great impact on the performance of routing protocol for DTN; high-speed nodes can deliver messages to a long-distance destination quickly.

Bus service is accessible by the node at predefined bus stops. Initially, the node would walk to the nearest bus station and wait for the bus; when the bus arrives, the node enters the bus and drops at the bus stop closest to its destination. The node switches to a walking submodel to complete its journey to the final destination.

The nodes in our model move on the map; this is another aspect of realism. The maps contain the homes, classes, cafeterias, playgrounds, shops, PoIs, and bus stops. The map data are essential for restricting the movement of the nodes to specific areas, which helps increase node localization. It is used to distribute nodes in the simulation area uniformly.

The EPOM model generates mobility patterns through intercluster and intracluster movements. Therefore, at each time instant, a node is either in intercluster or intracluster movement mode controlled by the two-step Markov model in Figure 10. When a node is in the intracluster movement mode, it explores the point of interest within its community and walks to the preferred POIs or generates a travel distance chosen from a lognormal distribution bounded by the community size.

The direction of movement is chosen from a bias direction symmetry distribution in the range $[0,2 \pi$ [; see Figure 2. The lognormal distribution of the intracluster

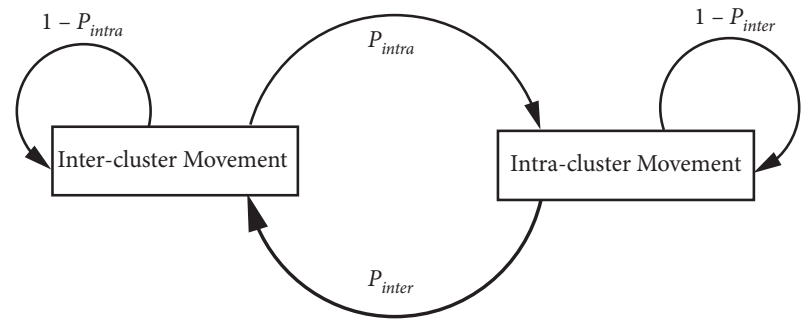

Figure 10: Two steps Markov model for switching between the intercluster and intracluster movements.

travel distance means nodes visit closer locations more frequently than distant locations.

4.7. Obstacle Submodel. The obstacle submodel describes how the EPOM model handles collision avoidance between nodes and other obstructing objects along their movement trajectories. In the case of static obstacles with zero speed, such as pedestrians standing on the road, at the middle of the corridors, or any other stationary object, we define the location of different obstacles on the map using OpenJUMP (http://openjump.org/) geographic information system program as in Figure 11.

The transport submodel moves the node from the current location (e.g., home) to the destination (e.g., class).

The Dijkstra shortest path algorithm calculates the shortest path from the current location to the destination. We have two scenarios here: in the first scenario, there is no obstacle on the path, while in the second scenario, an obstacle is encountered along the shortest path. In the first scenario, a node would follow the shortest path to its 


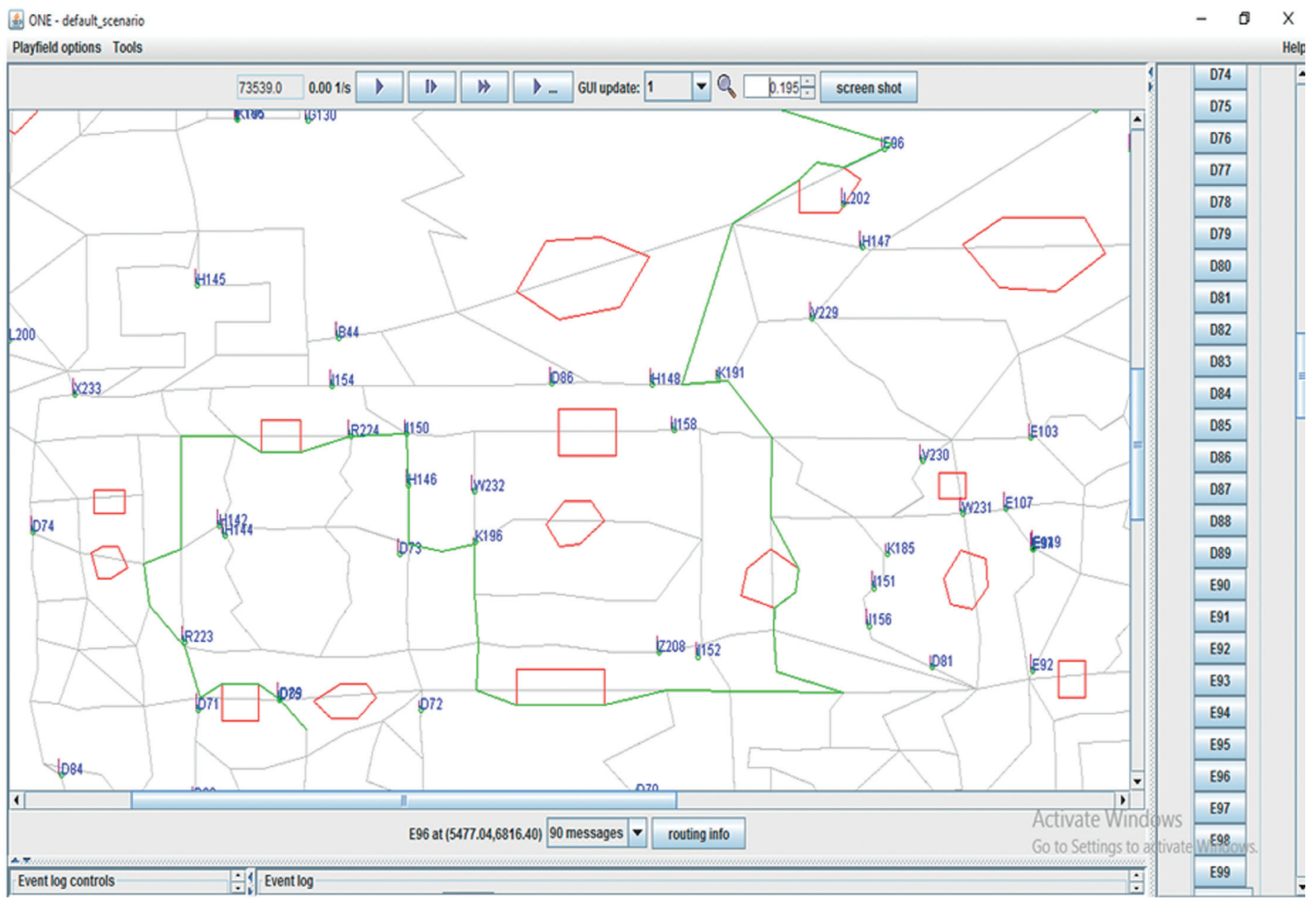

Figure 11: An example of visualization of a mobility scenario on the ONE simulator. The red irregular polygons mimic random obstacles. The blue numbered icons stand for nodes. The gray line stands for normal trajectories without obstacles, and the green line represents a new trajectory created by node E96 using the Escape path mobility model.

destination without obstruction, but in the second scenario, a node would explore the logic in Algorithm 2 to generate an escape path using the following transitions:

Algorithm 2 avoids collision with an obstacle by generating an escape path as shown in Figure 12. In Algorithm 2 , line 1 gets the coordinates of an obstacle's vertices $V=(\mathrm{A}, \mathrm{C}, \mathrm{D}, \mathrm{F}, \mathrm{E}, \mathrm{B})$. Note that the shape of an obstacle determines the number of vertices. In line 2 , a user finds the nearest vertex $\mathrm{A}$. It moves to vertex $\mathrm{A}$ in line 4 . It finds the neighbors of vertex A (i.e., $B$ and $C$ ) in line 5 and sets the next escape vertex to the nearest neighbor $B$ in line 6 . It then checks the condition in line 7; if the distance from its current location $\mathrm{A}$ to the destination is less than the distance from its neighbors $\mathrm{C}$ and $\mathrm{B}$ to the destination, it moves directly to the destination (line 8 ); otherwise, it returns to line 3.

Considering a human movement behavior of walking beside the edges of an obstructing body until it passes the section of the obstacle that blocks it, the algorithm behaves similarly by creating a path beside (not on the edges) the edges of the obstacle. Some existing works have proposed a Bezier curve [32] or branching to the closest neighbor node [28], which is not always realistic because a human path of escaping obstacle cannot always be curved, just like an isolated obstacle which may not have a closer neighbor.

\section{Model Implementation}

The Escape Path Obstacle-based Movement model was implemented on the Opportunistic Network Environment (ONE) simulator $[46,47]$ as a collection of different submodels. ONE supported different movement models such as the Random Waypoint Movement (RWP), Map-Based Movement (MBM), Shortest Path Map-Based Movement model (SPMBM), and Route-Based Movement model (RBM). MBM is a special type of RW in which nodes move along the map paths defined in Well-Known Text (WKT) files. We used the Open-JUMP Geographic Information System (GIS) program to define the location of obstacles, homes, classes, cafeterias, playgrounds, shops, and points of interest for off-campus activities. We created a main movement model that inherited the extended movement model of ONE and controlled the movement of nodes going to school, going to the cafeteria, going to sport, going shopping, or similar activities outside the campus, and finally returning home to sleep. The main model orders and switches between submodels, passes the control to the submodels responsible for different activities, facilitates the movement to the destination by giving information about the destination to the transport submodels, and decides on the probability to walk or use bus based on the setting configuration. 


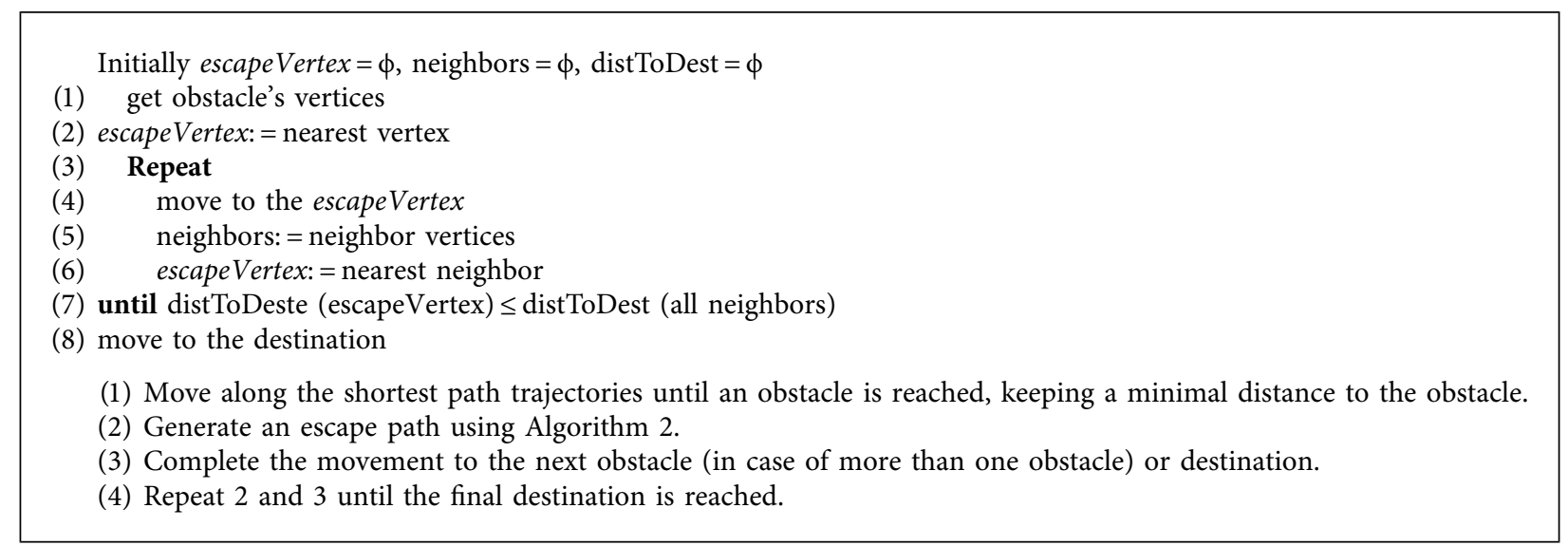

Algorithm 2: Escape path movement for node $i$.

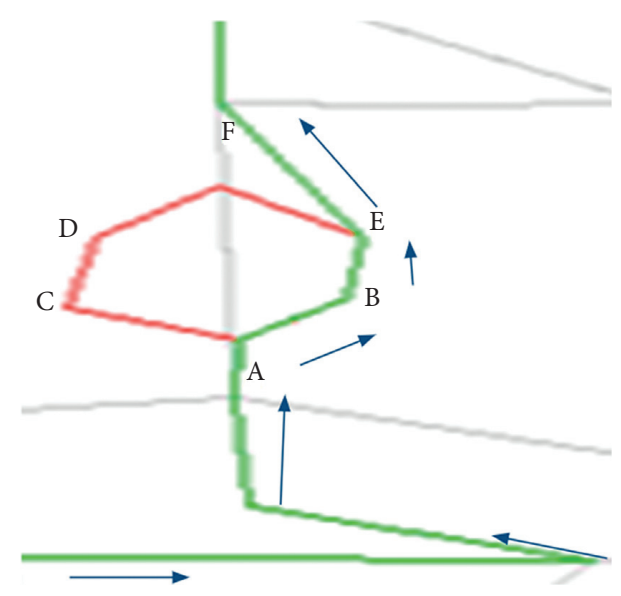

FIgURE 12: Escape path generated with Algorithm 2.

In a real scenario, an obstacle, such as the floor, walls, buildings, or mountains, exists and impacts mobility and signal attenuation. To reflect this impact, we modify the method "isWithinRange(DTNHost anotherHost)" for the class NetworkLayer of the ONE simulator to reflect the signal's attenuation in the propagation model. When a node's signal propagates through an obstacle, it suffers attenuation due to the effects of diffraction, reflection, and scattering. Some attenuation results [33] are presented in Table 4 . The attenuated values are randomly taken from a uniform distribution between 40 and $60 \mathrm{~dB}$, which reflects the fact that obstacles have at least double walls. A connection is created when the radio signal is greater than a fixed threshold (transmitting range).

\section{Validation}

Our goal is to show that our conceptual model (EPOM) is generic enough to be fine-tuned with a few parameters to show matching characteristics with the NCSU GPS traces [36], in terms of the spatial features: intracluster travel distance and intracluster direction of movement as well as the temporal feature (i.e., pause time). We also show that
EPOM connectivity features matched those of iMote real traces [44] in terms of contact duration and intercontact time distribution.

After the wake-up, a node starts to walk using the current mobility model; a node switches to different locations from the current location using the five steps Markov model depending on the time of the day. See Table 5 for the list of simulation parameters.

We simulate the random waypoint model on the same size simulation area with 1000 nodes uniformly distributed. Each node randomly chooses a waypoint and move with a speed of $0.5-5 \mathrm{~m} / \mathrm{s}$; when a node reaches the destination, it pauses for $1-3600 \mathrm{~s}$. Both the speed and pause time are uniformly distributed.

The simulation was run for the length of $T=5 \times 10^{5} \mathrm{~s}$, which is approximately five days. We assume all events are uniformly distributed over a longer period of time and consider the probability of an event of length $x, p(x)$. We record only events that begin and end within the observed interval. We create the Complementary Cumulative Density Function (CCDF, $P[X>x]$ ) for the distribution of contact duration, intercontact time, intertravel distance, intratravel distance, intracluster movement direction and pause time.

Settings: our simulation environment is a map of parts of the Université Paris Saclay campus, edited using OpenJUMP geographic information system program with 1000 nodes moving on the area of roughly $5000 \times 3000 \mathrm{~m}^{2}$. We created different WKT files for the map roads, homes, lecture rooms, cafeterias, sport, off-campus activities locations, PoIs, and obstacles.

Each node is assigned with a unique home located on the map as its starting point in the simulation, a wake-up time drawn from a normal distribution to each node.

6.1. Spatiotemporal Features. We start with the intracluster feature being one of the most important aspects of our study. We divide the main simulation domain into a number of equal size communities denoted by $c$ to account for the dynamic clusters. $C \epsilon\left\{1, \ldots, N_{c}\right\}$, where $N_{c}$ is the total 
TABLe 4: Power attenuation values.

\begin{tabular}{lcc}
\hline & Home & Office \\
\hline Single wall & $6-20 \mathrm{~dB}$ & $6-20 \mathrm{~dB}$ \\
Double wall & $40-50 \mathrm{~dB}$ & $50-60 \mathrm{~dB}$ \\
\hline
\end{tabular}

TABLE 5: Summary of the simulation parameters.

\begin{tabular}{lc}
\hline Parameter & Value \\
\hline Number of nodes & 1000 \\
Simulation length & $500,000 \mathrm{sec}$ \\
Transmit range & $10 \mathrm{mb}$ \\
Obstacle path transmit range & {$[5,10] \mathrm{mb}$} \\
World size & $5000 \times 3000 \mathrm{~m}^{2}$ \\
Walking speed & {$[1,3] \mathrm{m} / \mathrm{s}$} \\
Bus speed & {$[7,10] \mathrm{m} / \mathrm{s}$} \\
Transmit speed & $250 \mathrm{kbs}$ \\
Routing protocol & Epidemic \\
Interface type & Simple broadcast interface \\
Buffer size & $50 \mathrm{mb}$ \\
Message size & {$[500 \mathrm{~kb}, 1 \mathrm{mb}]$} \\
Message interval & {$[25,35] \mathrm{sec}$} \\
Message TTL & $1,430 \mathrm{sec}$ \\
\hline
\end{tabular}

number of communities in the domain. During our analysis, we find out that each walker is associated with an average of three dynamic clusters per day, as shown in Figure 13 depending on the degree of the repetitiveness of the user's schedule; we can exploit this type of temporal mobility feature to predict a possible user location. Similarly, it can be used by the opportunistic routing protocol to schedule package forwarding.

After tuning our model, it generates matching walking clusters with the KAIST data. Figure 14 shows one-day dynamic clusters of Node 4 generated from the EPOM model. The generated clusters matched with that of the KAIST trace in Figure 1 for the trace file sixteen [36].

Next, we focus on the intracluster travel distance to capture the neighborhood exploration observed in the real traces. In our model, at each time instant, a node is either in intercluster or intracluster movement mode managed by a two-step Markov model in Figure 15. When a node is in the intracluster movement mode, it explores the point of interest within its community and walks to the preferred PoIs or generates a travel distance uniformly chosen at random from a lognormal distribution bounded by the community size. The direction of movement is uniformly chosen at random from the bias symmetry distribution of the empirical data shown in Figure 2. The lognormal distribution of the intracluster travel distance means nodes visit closer locations more frequently than distant locations.

Figure 16 shows the distribution of direction angle generated from the synthetic traces of the EPOM model. The distribution is similar to the distribution of NCSU trace in Figure 2. The main take-home message from the two distributions is the movement within dynamic clusters which is not random but bias toward some PoIs and popular locations within the community.

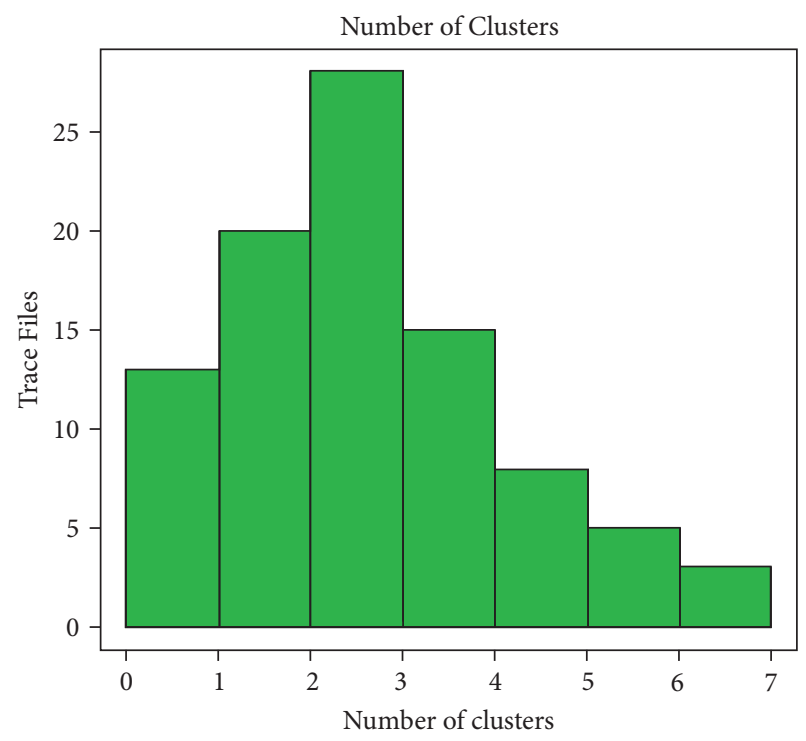

FIgURE 13: Number of dynamic clusters per trace file in KAIST traces.

Figure 17 shows an intracluster travel distance generated from our model compared to that of the empirical data. The two distributions are similar for a longer period of time but slightly differ at the tail.

This is a consequence of the size of the community in the simulation domain. Therefore, EPOM replicates intracluster travel distance as observed in the KAIST empirical data.

Next, we concentrate on the general domain by analyzing the intercluster travel distance distribution for the whole domain generated by the EPOM model and compare it with the empirical distribution observed from the 


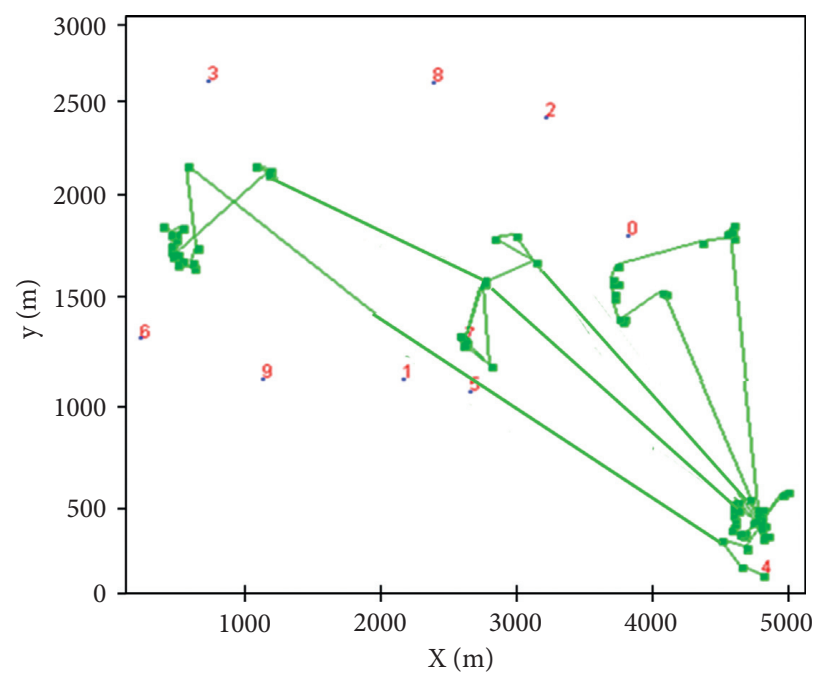

Mobile Nodes

Waypoints

FIgURE 14: The synthetic clusters generated by node four in the EPOM model. The red numbers represent mobile nodes, the green points indicate waypoints, and the green lines represent the node trajectories. We can see the waypoints as dynamic clusters.

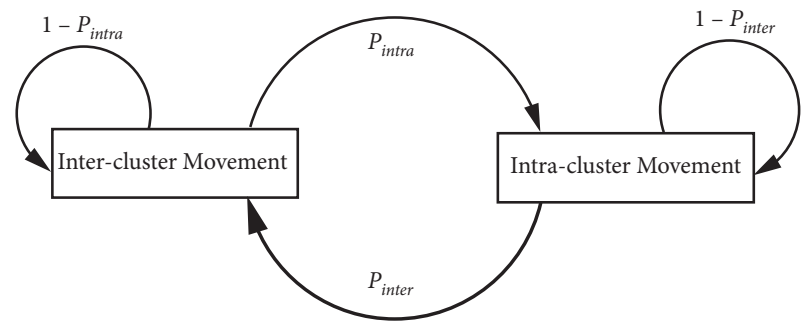

FIGURE 15: Two-state Markov model for intercluster and intracluster movement.

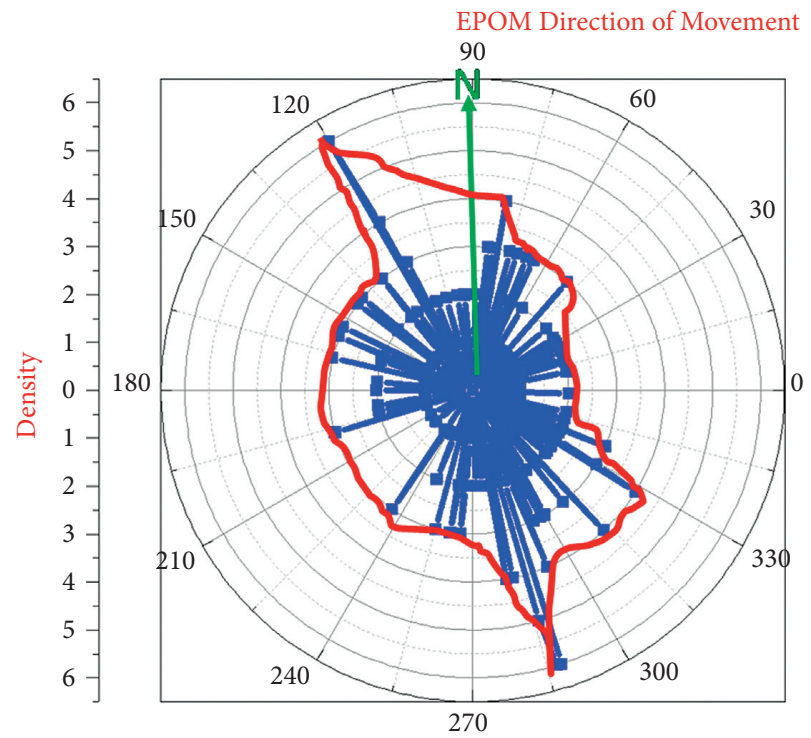

FIgURE 16: The bias symmetry distribution of direction angle for the EPOM clusters. The $x$-axis represents the angular (units are in degrees) and the $y$-axis is the density of movement toward a given direction. The bin size is $1^{\circ}$. Each direction is weighted by the duration of its movement.

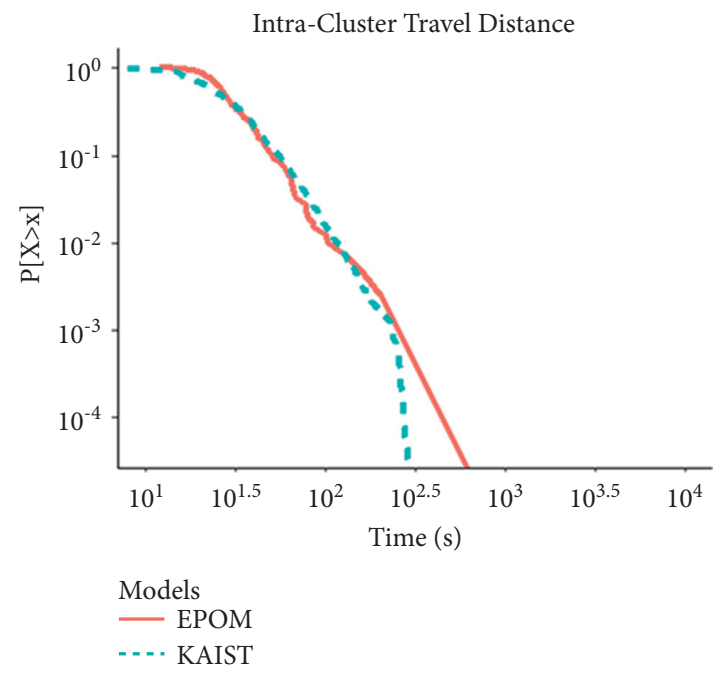

FIgURE 17: Intracluster travel distance for the EPOM and KAIST traces. Both curves follow a lognormal distribution, meaning people visit some preferred nearby locations more than far distant locations.

empirical data. This is the approach adopted by most of the existing works in $[48,49]$.

Figure 18 shows the intercluster travel distance distribution for the KAIST, EPOM, and RWP traces. The distribution of the EPOM and KAIST traces fits the truncated power-law distribution. It shows that users tend to undertake many short walks in a cluster and occasionally take long-distance walks. We also note that such short-distance walks that evolve over time are the consequence of intracluster movements. In contrast, the curve for the conventional RWP model fits uniform distribution, which does not differentiate between short and long walks. This feature does not resemble the realistic nature of human mobility patterns. 


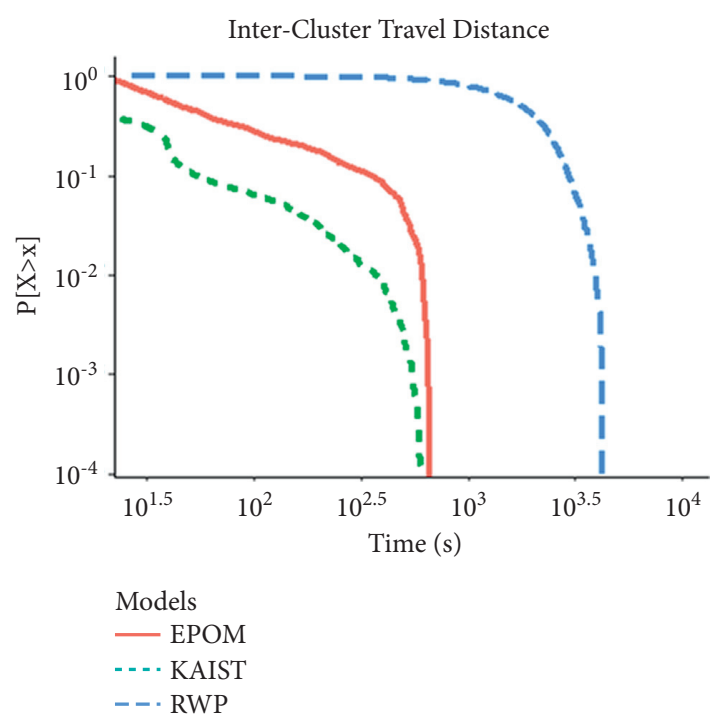

FIGURE 18: The intercluster travel distance for the EPOM, KAIST, and RWP models. The curves for the EPOM model and KAIST traces exhibit power-law decay for a long period, supporting the realistic nature of the human mobility pattern for taking short walks more than a long journey. The RWP curve is uniformly distributed and does not differentiate between short walks and long journeys.

Mobility temporal characteristics analysis of user's temporal locations at a certain period gives us an insight into the possibility of predicting users' location, how long a user could stay at a given location, that is, pause time, when the user is expected to return to a given location, that is, return time and why a user exhibits a skewed visiting behavior to some locations, that is, dynamic community walk.

We study the pause time distribution of the KAIST campus traces in [36] and tune the EPOM model to generate a pause time distribution similar to the empirical distribution observed. Figure 19 shows the pause time distribution of the KAIST trace and EPOM traces. The distribution is found to be power law with a heavy tail. This shows that students spent a long time at some locations, such as lecture rooms but stayed for a short time at most locations such as shopping malls and cafeterias. This distribution is consistent with the distribution of pause time observed in Dartmouth campus real traces in [23].

The fact that users pause for a long time at some preferred location also indicates that users predominantly take short walks within the community of such locations.

We observed that users are associated with an average of three dynamic clusters in one working day, which evolve over time, as shown in Figure 13. This fact is true for all users, except for stationary users.

6.2. Connectivity Features. In this section, we investigate how closely the EPOM model reproduces the distribution of the studied connectivity metrics as observed in the empirical data of the realistic traces in [44]. We compare the distribution generated by both EPOM, iMote traces, and Random Waypoint on each plot.

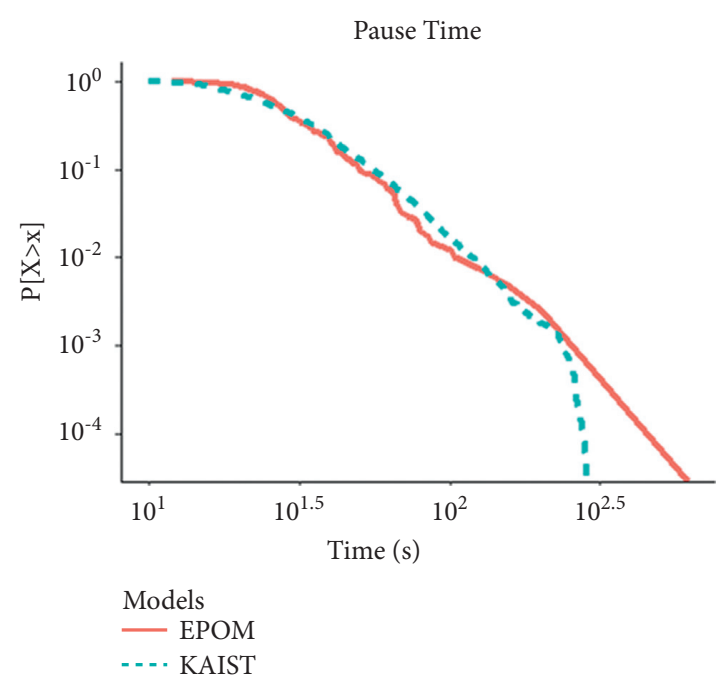

FIgURE 19: The pause time distribution of the KAIST and EPOM traces. The figure indicates that humans mostly stay short in most places they visit and stay at few locations.

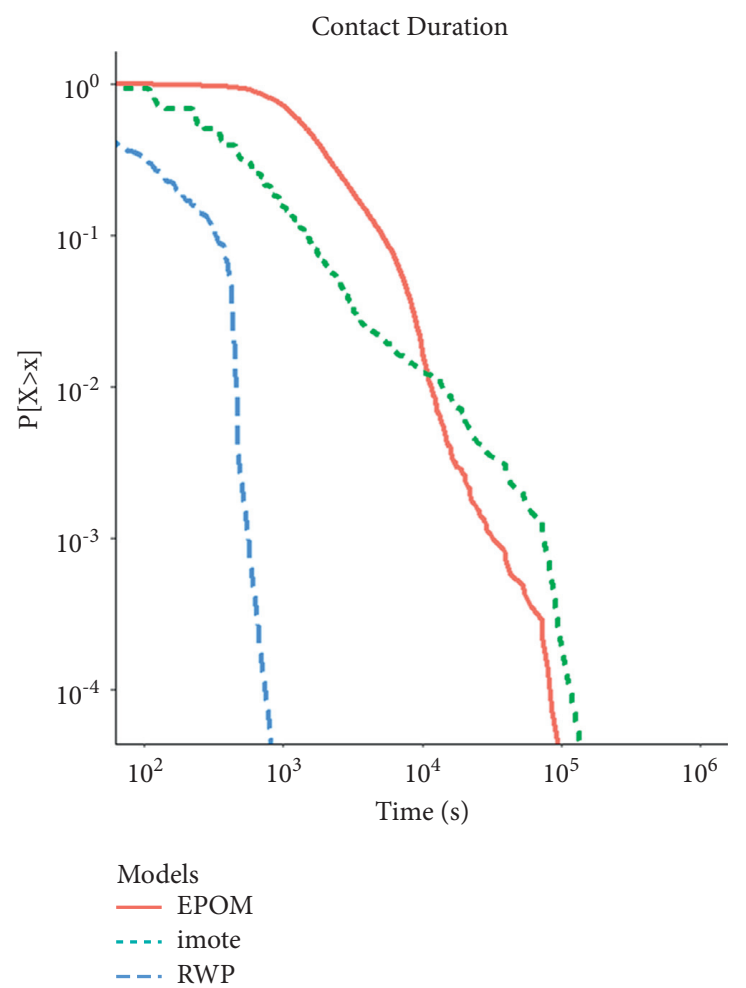

FIgure 20: The PDF of the contact time distribution for the EPOM, iMotes, and RWP model. The EPOM model follows power-law distribution for a long time, just like the iMotes traces, but RWP follows exponential distributions with very short contacts.

Figure 20 shows the aggregate distribution of contact duration for EPOM, iMote traces, and RWP. Each plot shows the complementary cumulative distribution function of a contact duration using a log-log scale. We see that the EPOM distribution follows power-law decay for a long time, similar to the distribution of iMotes traces. This is consistent 


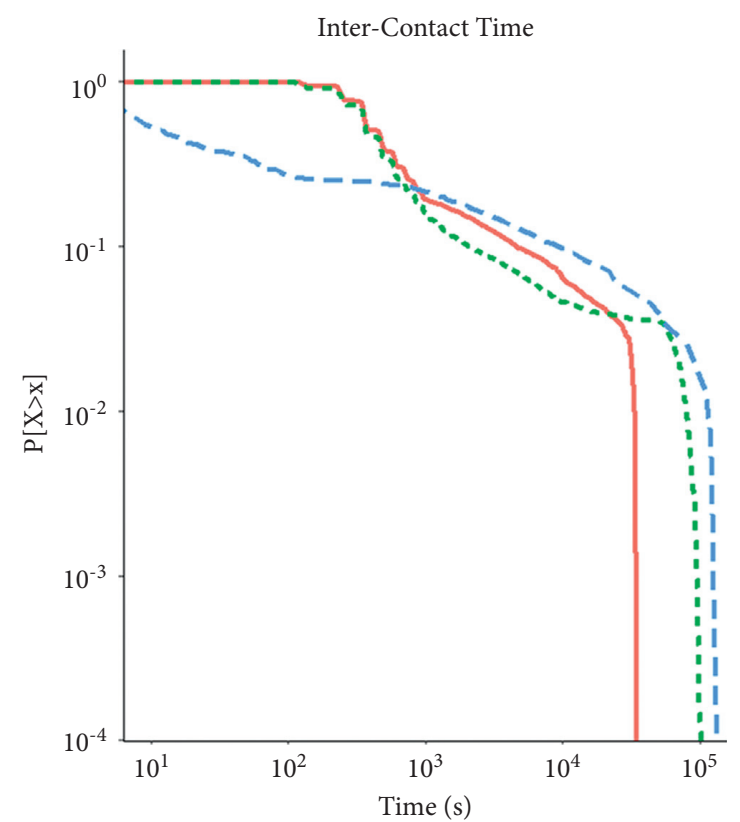

Models

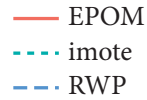

Figure 21: The PDF of the intercontact time distribution for the EPOM, iMotes, and RWP model. It shows that both the EPOM and iMote traces curves exhibit power-law decay with exponential cut-off, unlike RWP, which entirely follows an exponential distribution.

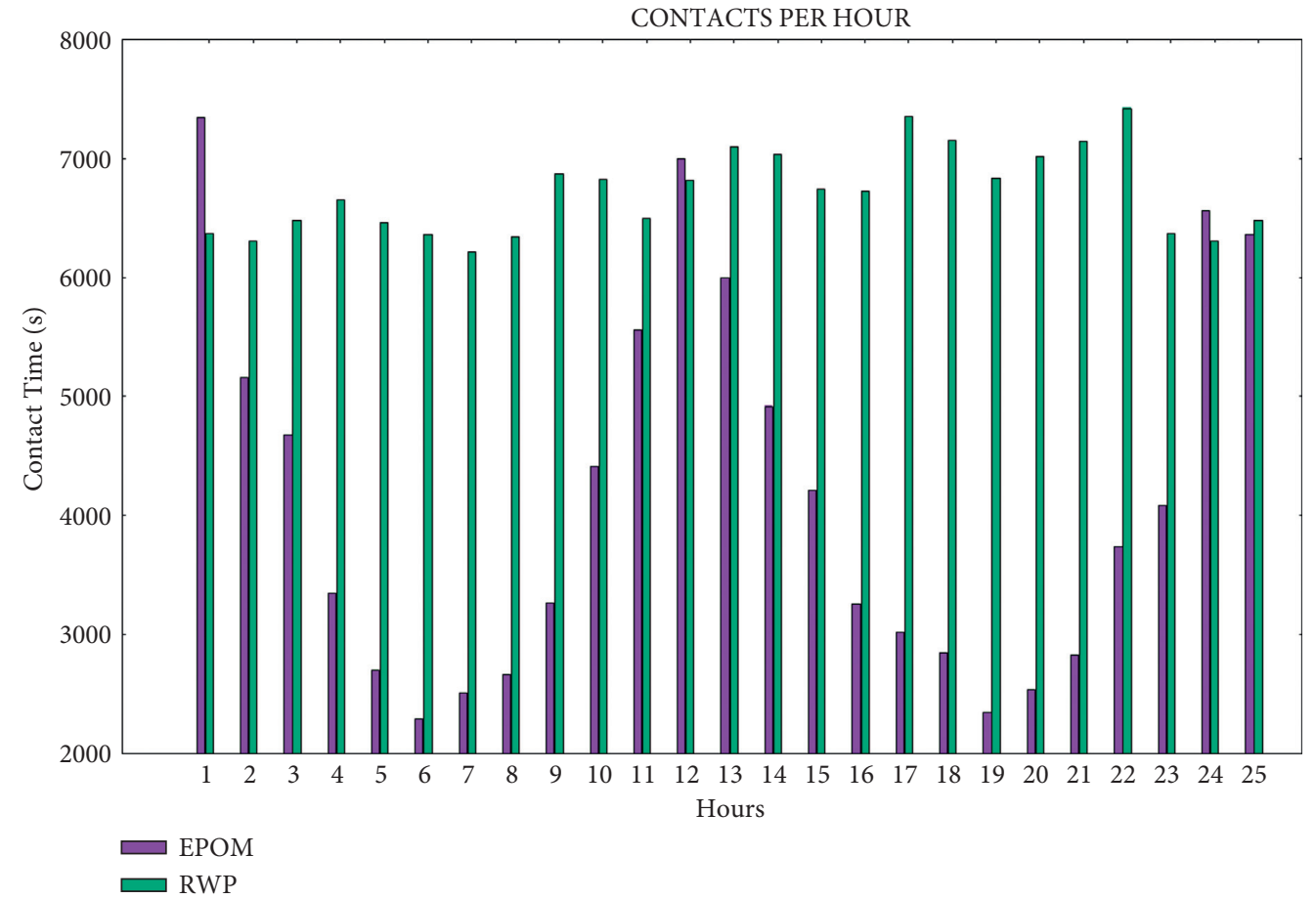

Figure 22: The distribution of contacts for each simulation hour. The EPOM model has shown repetitiveness of hourly activities, unlike RWP, which shows uniform distribution of activities for each hour. 
with the findings in most research on human mobility contact distribution [50]. The distribution of RWP consists of only a short time with exponential decay. The power-law feature of human mobility indicates that more nodes have contact opportunities for a shorter time while only a few nodes stay connected for a longer time. A DTN routing algorithm can be designed to exploit this feature in conjunction with the spatiotemporal features to decide the best way to route a message from the source to destination(s).

From Figure 21, we see the intercontact (ICTs) time distribution for EPOM, iMote traces, and RWP. Figure 21 shows that both the EPOM and iMote traces curves exhibit power-law decay with exponential cut-off, unlike RWP that entirely follows an exponential distribution. The distribution of ICTs for the EPOM is also consistent with the feature of the realistic ICTs discovered in [51]. The power-law nature of ICTs plays an important role in DTNs as it fundamentally impacts the behavior of networking protocols [51]. Though shorter intercontact time means more frequent connection, nodes with longer intercontact times are possibly assumed to have new data to share.

Figure 22 presents contacts for each simulation hour. Figure 22 shows the repetitiveness of hourly activities. We use 43200 seconds as working day length. The contact per hour RWP is uniform throughout the simulation. Observe the repetitive behavior of the EPOM, which captures students' daily routine activities at specific hours of the day.

\section{Conclusion and Future Work}

In this paper, we conduct an in-depth study of human mobility patterns using realistic datasets for Bluetooth encounters and Global Positioning System (GPS) track-logs traces at the finegrain level to better understand human mobility properties and uncover hidden patterns. Consequently, we have discovered time-varying human mobility patterns associated with a dynamic evolution of movement clusters. We proposed a new mobility model that mimics the realistic mobility patterns of real-world traces in the presence of obstacles of different shapes and sizes. The model describes various student activities that routinely evolve over time, such as going to the lectures, going to the cafeteria, sport, and shopping. We have shown that the model produces the distribution of the intercluster travel distance, intracluster travel distance, intracluster direction of movement, contact duration, intercontact time, and pause time, similar to the distribution of realistic traces.

For future work, we intend to extend the model to the urban scenario through an extensive study of dynamic clusters evolution of pedestrians and bus commuters. Consequently, we intend to design an efficient predicting framework for human mobility. The new framework will exploit the existing and new uncovered features to predict the user's subsequent displacement, stay duration, and possible contact.

\section{Data Availability}

All data and models that support the findings of this study are available upon reasonable request.

\section{Conflicts of Interest}

The authors declare no conflicts of interest.

\section{Acknowledgments}

This work was financially supported by the Petroleum Technology Development Funds of Nigeria (Grant no. PTDF/ED/PHD/SPA/76/18). Moreover, a part of the manuscript has been published at a conference in [52].

\section{References}

[1] K. Ahmad, N. I. Udzir, and G. C. Deka, Opportunistic Networks, Chapman and Hall/CRC, Boca Raton, Florid, Oct. 2018.

[2] K. Fall, "A delay-tolerant network architecture for challenged internets," in Proceedings of the conference on Applications, technologies, architectures, and protocols for computer communications - SIG-COMM 03, pp. 27-34, ACM Press, Karlsruhe Germany, 25 August 2003.

[3] G. F. Lawler and V. Limic, Random Walk: A Modern Introduction, Cambridge University Press, Cambridge, England, 2009.

[4] A. Clementi, A. Monti, F. Pasquale, and R. Silvestri, "Information spreading in stationary Markovian evolving graphs," IEEE Transactions on Parallel and Distributed Systems, vol. 22, no. 9, pp. 1425-1432, Sep. 2011.

[5] B. B. Mandelbrot, The Fractal Geometry of Nature/Revised and Enlarged Edition, W. H. Freeman, vol. 495, p. 01, New York, US, 1983.

[6] K. Lee, S. Hong, S. J. Kim, I. Rhee, and S. Chong, "SLAW: a new mobility model for human walks," in Proceedings of the IEEE INFOCOM - The 28th Conference on Computer Communications, IEEE, Rio de Janeiro, Brazil, 19 April 2009.

[7] A. Munjal, T. Camp, and W. C. Navidi, "Smooth," in Proceedings of the 14th ACM international conference on Modeling, analysis and simulation of wireless and mobile systems - MSWiM, vol. 11, ACM, Miami Florida USA, 31 October 2011.

[8] D. B. Johnson and D. A. Maltz, "Dynamic source routing in ad hoc wireless networks," in The Kluwer International Series in Engineering and Computer Science, pp. 153-181, Springer US, Boston, MA, 2001.

[9] J. Yoon, M. Liu, and B. Noble, "Random waypoint considered harmful," in Proceedings of the Twenty-second Annual Joint Conference of the IEEE Computer and Communications Societies (IEEE Cat. No.03CH37428), IEEE, San Francisco, CA, USA, 30 March 2003.

[10] Z. Haas, "A new routing protocol for the reconfigurable wireless networks," in Proceedings of the ICUPC 97 - 6th International Conference on Universal Personal Communications, IEEE, San Diego, CA, USA, 12 October 1997.

[11] M. Moussaïd, D. Helbing, S. Garnier, A. Johansson, M. Combe, and G. Theraulaz, "Experimental study of the behavioural mechanisms underlying self-organization in human crowds," Proceedings. Biological sciences, vol. 276, no. 1668, pp. 2755-2762, May 2009.

[12] M. Moussaid, D. Helbing, and G. Theraulaz, "How simple rules determine pedestrian behavior and crowd disasters," Proceedings of the National Academy of Sciences, vol. 108, no. 17, pp. 6884-6888, Apr. 2011.

[13] M. Haghani and M. Sarvi, "Simulating pedestrian flow through narrow exits," Physics Letters A, vol. 383, no. 2-3, pp. 110-120, Jan. 2019. 
[14] J. P. Dominguez-Morales, A. Rios-Navarro, M. DominguezMorales et al., "Wireless sensor network for wildlife tracking and behavior classification of animals in doñana," IEEE Communications Letters, vol. 20, no. 12, pp. 2534-2537, Dec. 2016.

[15] M. Sanchez, "Mobility models," 1998, http://www.disca.upv. es/misan/mobmodel.htm.

[16] J. Broch, D. A. Maltz, D. B. Johnson, Y.-C. Hu, and J. Jetcheva, "A performance comparison of multi-hop wireless ad hoc network routing protocols," In Proceedings of the 4th Annual ACM/IEEE International Conference on Mobile Computing and Networking - MobiCom, vol. 98, 1998.

[17] C.-C. Chiang and M. Gerla, "On-demand multi-cast in mobile wireless networks," in Proceedings of the Sixth International Conference on Network Protocols (Cat. No.98TB100256), IEEE Comput. Soc, Austin, TX, USA, 13 October 1998.

[18] F. Bai, N. Sadagopan, and A. Helmy, "IMPORTANT: a framework to systematically analyze the impact of mobility on performance of routing protocols for adhoc networks," in Proceedings of the Twenty-second Annual Joint Conference of the IEEE Computer and Communications Societies (IEEE Cat. No.03CH37428), IEEE, San Francisco, CA, USA, 30 March 2003.

[19] F. Ekman, A. Keränen, J. Karvo, and J. Ott, "Working day movement model," in Proceedings of the 1st ACM SIGMOBILE workshop on Mobility models - MobilityModels, vol. 08, Hong Kong, China, 26 May 2008.

[20] X. Zhu, Y. Bai, W. Yang, Y. Peng, and C. Bi, "SAME: a students daily activity mobility model for campus delaytolerant networks," in Proceedings of the 18th Asia-Pacific Conference on Communications (APCC), IEEE, Jeju, Korea (South), 15 October 2012.

[21] D. Kotz and K. Essien, "Analysis of a campus-wide wireless network," in Proceedings of the 8th Annual International Conference on Mobile Computing and Networking - MobiCom 02, ACM Press, 2002.

[22] P. Hui, A. Chaintreau, J. Scott, R. Gass, J. Crowcroft, and C. Diot, Pocket Switched Net-Works: Real-World Mobility and its Consequences for Opportunistic Forwarding, University of Cambridge, Cambridge, England, August 2005.

[23] M. Kim, D. Kotz, and S. Kim, "Extracting a mobility model from real user traces," in Proceedings of the International Conference on Computer Communications, IEEE, Barcelona, Spain, 23 April 2006.

[24] D. Hrabcak, M. Matis, L. Dobos, and J. Papaj, "Students social based mobility model for MANET-DTN networks," Mobile Information Systems, vol. 2017, pp. 1-13, Article ID 2714595, 2017.

[25] J. Topping, "Investigations on the theory of the brownian movement," Physics Bulletin, vol. 7, no. 10, p. 281, oct 1956.

[26] E. Royer, P. Melliar-Smith, and L. Moser, "An analysis of the optimum node density for ad hoc mobile networks," in Proceedings of the IEEE International Conference on Communications. Conference Record (Cat. No.01CH37240), IEEE, Helsinki, Finland, 11 June 2001.

[27] M. McNett and G. M. Voelker, "Access and mobility of wireless PDA users," ACM SIGMOBILE - Mobile Computing and Communications Review, vol. 9, no. 2, pp. 40-55, Apr. 2005.

[28] D. Wu, J. Li, and J. Liu, "A random obstacle-based mobility model for delay-tolerant networking," International Journal of Network Management, vol. 21, no. 4, pp. 326-339, Jul. 2011.

[29] Y. Wang, X. Zhou, S. Zhang, and W. Huang, "Human mobility model in mission-oriented opportunistic networks," in
Proceedings of the 7th IEEE Intl Conference on Big Data Security on Cloud (BigDataSecurity), IEEE Intl Conference on High Performance and Smart Computing, (HPSC) and IEEE Intl Conference on Intelligent Data and Security (IDS), pp. 202-207, IEEE, NY, USA, 15 May 2021.

[30] M. Musolesi, S. Hailes, and C. Mascolo, "An ad hoc mobility model founded on social network theory," in Proceedings of the 7th ACM international symposium on Modeling, analysis and simulation of wireless and mobile systems - MSWiM 04, ACM, Venice Italy, 4 October 2004.

[31] K. Herrmann, "Modeling the sociological aspects of mobility in ad hoc networks," in Proceedings of the 6th international workshop on Modeling analysis and simulation of wireless and mobile systems - MSWIM 03, ACM Press, San Diego CA USA, 19 September 2003.

[32] W. Wang, J. Wang, M. Wang, B. Wang, and W. Zhang, "A realistic mobility model with irregular obstacle constraints for mobile ad hoc networks," Wireless Networks, vol. 25, no. 2, pp. 487-506, Sep. 2017.

[33] A. Jardosh, E. Belding-Royer, K. Almeroth, and S. Suri, "Realworld environment models for mobile network evaluation," IEEE Journal on Selected Areas in Communications, vol. 23, no. 3, pp. 622-632, 2005.

[34] C. Papageorgiou, K. Birkos, T. Dagiuklas, and S. Kotsopoulos, "An obstacle-aware human mobility model for ad hoc networks," in Proceedings of the 2009 IEEE International Symposium on Modeling, Analysis \&amp; Simulation of Computer and Telecommunication Systems, IEEE, London, UK, 21 September 2009.

[35] D. Kotz, T. Henderson, I. Abyzov, and J. Yeo, "CRAWDAD dataset dartmouth/campus (v. 2009-09-09),” 2009, https:// crawdad.org/dartmouth/campus/20090909.

[36] I. Rhee, M. Shin, S. Hong, K. Lee, S. Kim, and S. Chong, Crawdad Dataset Ncsu/mobilitymodels (V. 2009-07-23), 2009.

[37] D. Duong and S. Yoon, "A human mobility model in shipyards," In International Journal of Internet, Broadcasting and Communication, vol. 12, no. 4, pp. 93-101, 2020.

[38] S. Ghosh, S. K. Ghosh, and R. Buyya, "MARIO: a spatiotemporal data mining framework on google cloud to explore mobility dynamics from taxi trajectories," Journal of Network and Computer Applications, vol. 164, Article ID 102692, aug 2020.

[39] M. Yue, Y. Li, H. Yang, R. Ahuja, Y. Chiang, and C. Shahabi, "Detect: deep trajectory clustering for mobility-behavior analysis," in Proceedings of the 2019 IEEE International Conference on Big Data (Big Data), pp. 988-997, Atlanta, GA, USA, 10 December 2020.

[40] M. A. Rahman, N. Zaman, A. T. Asyhari, F. Al-Turjman, M. Z. A. Bhuiyan, and M. Zolkipli, "Data-driven dynamic clustering framework for mitigating the adverse economic impact of covid-19 lockdown practices," Sustainable Cities and Society, vol. 62, Article ID 102372, nov 2020.

[41] W. Hsu, T. Spyropoulos, K. Psounis, and A. Helmy, "Modeling time-variant user mobility in wireless mobile networks," in Proceedings of the 26th International Conference on Computer Communications, IEEE, Anchorage, AK, USA, 6 May 2007.

[42] B. A. A. Nunes, "Characterizing user mobility in wireless networks," California, US, Ph.D. dissertation, University of California Santa Cruz, School of Engineering.

[43] T. Henderson, "Project page of the crawdad," 2020, http:// crawdad.org/.

[44] J. Scott, R. Gass, J. Crowcroft, P. Hui, C. Diot, and A. Chaintreau, "CRAWDAD dataset cambridge/haggle (v. 
2009-05-29)," May 2009, https://crawdad.org/cambridge/ haggle/20090529/imote.

[45] S. C. Johnson, "Hierarchical clustering schemes," Psychometrika, vol. 32, no. 3, pp. 241-254, Sep. 1967.

[46] A. Keränen, J. Ott, and T. Kärkkäinen, "The ONE simulator for DTN protocol evaluation," in Proceedings of the SIMUTools '09: Proceedings of the 2nd International Conference on Simulation Tools and Techniques, ACM, Rome Italy, 2 March 2009.

[47] Tkk/Comnet, "Project page of the one simulator," 2013, http://www.netlab.tkk.fi/tutkimus/dtn/theone.

[48] A. Eitan, G. Robin, and N. Philippe, "Relaying in mobile ad hoc networks: the brownian motion mobility model," INRIA, Tech. Rep. RR-5311, Sep. 2004.

[49] M. C. González, C. A. Hidalgo, and A.-L. Barabási, "Understanding individual human mobility patterns," Nature, vol. 453, no. 7196, pp. 779-782, Jun. 2008.

[50] P. Pirozmand, G. Wu, B. Jedari, and F. Xia, "Human mobility in opportunistic networks: characteristics, models and prediction methods," Journal of Network and Computer Applications, vol. 42, pp. 45-58, 2014.

[51] A. Chaintreau, P. Hui, J. Crowcroft, C. Diot, R. Gass, and J. Scott, "Impact of human mobility on opportunistic forwarding algorithms," IEEE Transactions on Mobile Computing, vol. 6, no. 6, pp. 606-620, Jun. 2007.

[52] S. A. Bakura, A. Lambert, and T. Nowak, "An obstacle-aware mobility model for campus delay tolerant network," in Proceedings of the 2020 Zooming Innovation in Consumer Technologies Conference (ZINC), pp. 82-86, IEEE, Novi Sad, Serbia, 26 May 2020. 